\title{
Recent advances in understanding molecular mechanisms of primary afferent activation
}

\section{J N Wood}

Gut 2004;53(Suppl II):ii9-ii12. doi: 10.1136/gut.2003.033423

Thermal, mechanical, and chemical stimuli depolarise specialised damage sensing neurons to initiate electrical signals that may ultimately result in a sensation of pain. Over the past decade many of the receptors that transduce these signals have been identified by molecular cloning. In the absence of specific blockers, null mutant mice have proved valuable in exploring the function of these specialised receptors. As well as the mechanisms of signal transduction, the setting of thresholds for excitation and the transmission of electrical signals have also been the focus of intense interest. In vitro studies of dorsal root ganglion sensory neurons have thus facilitated rapid advances in our understanding of the biology of nociceptors. However, the specific properties of visceral afferents are poorly defined, and useful animal models of visceral pain are only now being developed. Visceral neuron receptor subtypes and the consequences of their activation in terms of pain perception and behaviour are thus subjects that still demand a major research effort.

Correspondence to: J N Wood, Molecular Nociception Group, Department of Biology, University College, London WCIE 6BT, UK;

J.Wood@ucl.ac.uk

Accepted for publication 16 October 2003
$\mathrm{T}$ he neurons present in the dorsal root ganglia of rodents are probably the most extensively studied of all mammalian neuronal subtypes. ${ }^{1}$ The functional diversity of these cells has been correlated with a variety of markers, including cell surface molecules, neurotransmitters, and cytoskeletal proteins. Damage sensing small diameter sensory neurons fall into two roughly equal populations with distinct neurotrophin requirements. About half of these express the high and low affinity NGF receptors (TrkA and p75 respectively), whereas a separate population, which can be surface labelled with isolectin B4 (IB4), are TrkA and p75 negative and have a trophic requirement for glial derived neurotrophic factors (GDNF). Three other GDNF related factors have been identified. These proteins, named persephin, neurturin, and artemin, signal through a receptor complex comprising the tyrosine kinase receptor c-ret, associated with TGF- $\beta$ receptor-like subunits that define the specificity of ligand binding.

The central terminals of these two classes of nociceptor are found in different areas of lamina II of the spinal cord. The GDNF sensitive population terminate in inner lamina II in the rat. However, the functional significance of this organisational difference is as yet unknown.
There seems to be a high level of expression of TrkA, the NGF receptor, in visceral afferents, but a specific visceral marker that would enable individual DRG neurons that innervate the viscera to be identified remains unknown. Central imaging studies in man have shown that, unlike somatic afferents whose activation results in a clear activation of the Sl somatosensory cortex, visceral sensory activation is primarily represented in the secondary somatosensory cortex, implying quite different pathways for the processing of visceral information that may begin with specialised subsets of visceral sensory neurons. In this review, we focus on some of the mediators and their receptors that are known to evoke both visceral and somatic pain.

\section{ACTIVATION OF PRIMARY SENSORY NEURONS \\ (1) Heat}

Noxious heat causes an inward current in a subset of nociceptive neurons with a threshold of activation of about $42^{\circ} \mathrm{C}$. This effect is mediated through a cation selective ion channel that is also gated by capsaicin, the pungent ingredient of red peppers cloned by the Julius laboratory. ${ }^{2}$ This observation provides a mechanism for the apparent sensation of heat generated by eating chilli peppers. Using a rat DRG cDNA library in a shuttle vector, pools of clones that conferred a response to capsaicin were isolated and syb selected. Increases in intracellular calcium in HEK293 cells transfected with defined pools of cDNA clones were measured using the calcium sensitive Fura-2 dye. The functional receptor TRP-vanilloid receptor-1 (TRPVI) resembles members of the "TRP" (transient receptor potential) family of proteins in terms of topological organisation. TRP proteins are 6-transmembrane calcium channels first identified in drosophila in the transient receptor potential (that is, TRP) mutants which show deficits in photoreception.

Abbreviations: NGF, nerve growth factor; GDNF, glial derived neurotrophic factors; GFR, growth factor receptor; GPI, glycophosphoinositol; IB4, isolectin B4; TrkA, tyrosine kinase A; DRASIC, DRG sensory neurons; TRP, transient receptor potential; TRPVI, TRP-vanilloid receptor 1; CMR1, cold/menthol receptor; ASIC, acid sensing ion channel; ENac, epithelial sodium channel; MDEG, mammalian degenerin; CNS, central nervous system; UTRs, un-translated regions; ATP, adenosine triphosphate; PPK, pickpocket; RPK, ripped pocket; VGSC, voltage gated sodium channels; TTX, tetrodotoxin, Cre-lox-p, cyclase recombinase locus of crossover. 


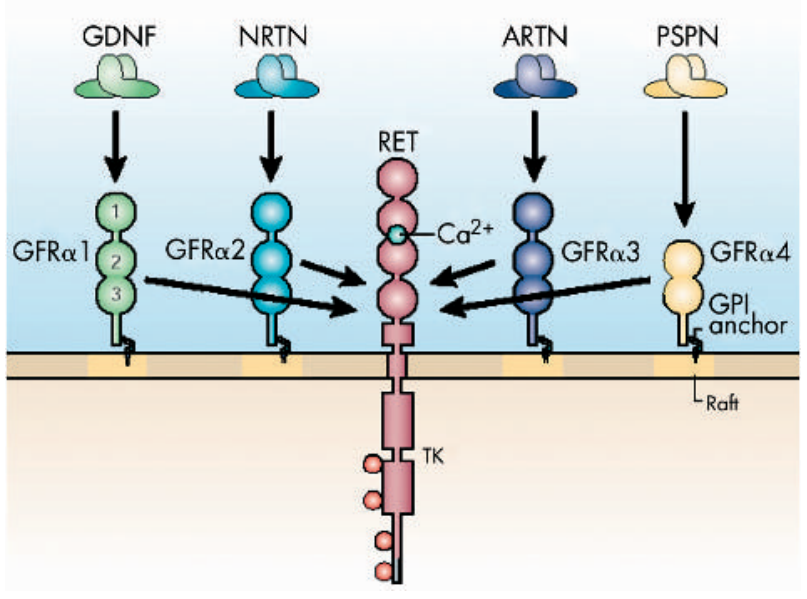

Figure 1 Cell signalling through the red tyrosine kinase by members of the glial derived neurotrophic factor family. The associated $\alpha$ subunits determine the specificity of the interaction between the various ligands $\operatorname{GDNF}(\alpha-1)$, neurturin $(\alpha-2)$, artemin $(\alpha-3)$, and persephin $(\alpha-4)$. GFR, growth factor receptor; GPI, glycophosphoinositol.

The existence of other TRPVl-like receptors and the large number of TRP family members raises the possibility that members of this channel family may be gated by a variety of different stimuli. Indeed, TRP family members have been shown to be involved in transducing a variety of extracellular signals, including light, heat, cold, and mechanical distension; intracellular mediators such as diacyl-glycerol and arachidonic acid metabolites, some acting through kinases may also activate some TRP channels. Recently, the TRPM8 channel has been shown to transduce noxious cold stimuli. ${ }^{3}$ Two other TRPv channels are gated by noxious heat, although they are not activated by capsaicin. All these channels are found in DRG sensory neurons. ${ }^{4}$ Interestingly, in birds, TRPVI is not activated by capsaicin, although its ability to detect heat and low $\mathrm{pH}$ is conserved. Julius et al have identified a region in rat TRPVI that confers capsaicin sensitivity to the chicken orthologue, and argue that this region is maintained in mammals because of the functional significance of activation by anandemide at the same site in mammals. Anandemide, an arachidonic acid derivative, was originally considered to be an endogenous cannibinoid-like ligand, acting only at cannibinoid $\mathrm{CB} 1$ and $\mathrm{CB} 2$ receptors. Bradykinin, a hyperalgesic mediator can also gate TRP channels via intracellular second messengers. Homology cloning and expression studies of VRl in combination with other TRP channels, and their regulation by $G$ protein coupled receptors should provide interesting evidence, not only on mechanisms of nociception but also for the normal gating mechanisms of TRP channels. TRP channels have been shown to heteromultimerise and to interact with other regulatory proteins. Recent studies describe the multimerisation of TRPVI to form mainly tetramers. Therefore, it seems plausible that different members of the TRPV receptor family may heteromultimerise, forming channels with new pharmacological characteristics, adding to the subtlety of signalling mechanisms present in primary sensory neurons.

\section{(2) Cold}

Using either bioinformatic tools or expression cloning using calcium imaging (a similar strategy to that which was used to identify the capsaicin receptor), two groups have identified a member of the transient receptor potential family (TRP) that is responsive to cold temperatures, as well as being activated by menthol. ${ }^{3}$ This receptor is known as CMRl (cold/menthol receptor) or TRPM8. The distribution of the receptor in sensory neurons is not disputed, but one group claims significant co-expression with heat receptors, whereas the other finds no overlap between TRPM8 and VR-1. TRPM8 is also expressed in non-sensory systems, for example epithelial cells in the trachea and prostate gland. It remains possible that other stimuli may activate TRPM8, in the same way that TRPVI is activated not only by noxious heat, but also protons and a variety of lipid ligands. Very recently another TRP channel activated by more extreme cold temperatures has also been identified in nociceptive sensory neurons. ${ }^{5}$

\section{(3) Low pH}

Damaged tissue is associated with lower extracellular pH. Protons are known to activate TRPVl and cause pain. However, other proton gated channels are also present within the peripheral nervous system. A proton gated cation channel named ASIC (acid sensing ion channel) expressed throughout the nervous system was the first channel of this class to be identified. The ASIC channels comprise a two transmembrane subunit that place them in the family of amiloride sensitive ENac (epithelial sodium channel) channels. ${ }^{6}$ The family has expanded to include the mammalian degenerin (MDEG) channels, renamed ASIC2a and 2b, and a related clone expressed in DRG sensory neurons named DRASIC or ASIC-3. Interestingly, all the ASIC clones have highly conserved extracellular cysteine residues, suggesting that the overall topology of the extracellular domain is similar within this particular class of receptor. Recent studies have provided compelling evidence that the members of the ENac family are tetramers, when heterologously expressed, in contrast to the trimeric or hexameric $\mathrm{P} 2 \mathrm{X}$ receptors.

Using homology cloning techniques, the ASIC homologue (DRASIC) was found to be present in sensory neurons. DRASIC is predominantly expressed in sensory neurons, but there is now some evidence that it is expressed in spinal cord and other CNS locations at lower levels. In addition, two splice variants of an additional channel named mammalian degenerin homologue-1 (MDEG-1) have been identified. MDEG- 1 is a functional proton-gated ion channel, but MDEG-2 is a splice variant that does not function except as a heteromultimer with ASIC or DRASIC. Interestingly, the properties of DRASIC/MDEG-2 heteromultimers are distinct from DRASIC, with altered kinetics and ionic permeabilities, providing strong evidence that members of the ENaC family may form heteromultimers of, presumably, four subunits in total. MDEG-1 and MDEG-2 are also known as ASIC2a and ASIC2b. ASIC2b, but not ASIC2a, is found in sensory neurons.

An ASIC related clone with distinct $5^{\prime}$ and $3^{\prime}$ untranslated regions (UTRs), named ASIC- $\alpha$, and a splice variant with a unique $5^{\prime}$ region and the same $C$ terminal and $3^{\prime}$ UTR as ASIC- $\alpha$ named ASIC- $\beta$, have also been recently identified by homology cloning. ASIC- $\beta$ has a unique 172 amino acid $N$ terminal region with a putative first transmembrane domain that is homologous to DRASIC channel sequence. A single ASIC- $\alpha 3.2 \mathrm{~kb}$ transcript is expressed in sensory neurons and other tissues. A similar sized transcript is expressed by ASIC$\beta$ in DRG alone, and is present only in a subset of small diameter sensory neurons ${ }^{6}$

\section{MECHANICAL STIMULATION}

Mechanical stimulation is clearly important in visceral pain processing. There is clear evidence that $\mathrm{C}$ fibres can be activated mechanically, suggesting that receptors are present on the sensory neurons themselves. Of the mechanosensitive channels defined by genetic studies of $C$ elegans and Drosophila melanogaster, many fall into the class of two transmembrane receptors exemplified by the epithelial sodium channel 
(ENac)/Degenerin family. ATP gated channels have also been implicated in mechanotransduction in mammals. Two types of receptor exist. P2X receptors are ATP gated ion channels, whereas the P2Y receptors are G protein coupled seven transmembrane receptors that act through second messenger cascades. Expression cloning of rat mechanoreceptors in Xenopus oocytes suggested that ATP, released through mechanical distortion, may activate the $\mathrm{P} 2 \mathrm{Yl}$ receptor present on large diameter sensory neurons, ${ }^{7}$ whereas more active mechanical distortion causes activation of the P2X3 receptor (also a two transmembrane ion channel). A variety of mechanisms for mechanosensation may thus exist. Two novel drosophila DEG/ENaC proteins, pickpocket (PPK) and ripped pocket (RPK), appear to be ion channel subunits. Amiloride and gadolinium, which block mechanosensation in vivo, inhibited RPK channels. Although PPK does not form channels on its own, it associates with and reduces the current generated by a related human brain $\mathrm{Na}+$ channel. The vertebrate homologues of these channels are thus candidate mechanoreceptors.

The channels that underlie mechano sensation remain unknown, although ASIC cation channels in the DEG/ENaC family have been proposed to detect cutaneous stimuli in mammals. DRASIC is present in several different specialised sensory nerve endings of skin, suggesting that it might participate in mechanosensation and/or acid evoked nociception. Disrupting the mouse DRASIC gene increased the sensitivity of mechanoreceptors detecting light touch, but it reduced the sensitivity of a mechanoreceptor responding to noxious pinch and decreased the response of acid and noxious heat sensitive nociceptors. ${ }^{8}$ The data support the view that DRASIC subunits participate in heteromultimeric channel complexes in sensory neurons. One way to directly identify mechanosensitive channels is to expression clone them after characterising them in primary cultures of sensory neurons

Mechanical stimulation of the somata of cultured rat DRG evokes inward cationic currents that display distinct properties between different subsets of neurons. The nociceptor population, defined by sensitivity to capsaicin, shows higher thresholds for the induction of an inward current and lower peak currents, than other mechanosensitive neurons. Extracellular calcium attenuates mechanosensitive currents to a greater degree in capsaicin insensitive neurons than capsaicin sensitive neurons. A subset of capsaicin sensitive IB4 positive sensory neurons was refractory to mechanical stimulation. All mechanosensitive currents were blocked by gadolinium $\left(\mathrm{IC}_{50} \approx 8 \mu \mathrm{M}\right)$ and ruthenium red $\left(\mathrm{IC}_{50} \approx 3 \mu \mathrm{M}\right)$. Disruption of the actin cytoskeleton by application of $10 \mu \mathrm{M}$ cytochalasin B blocked mechanosensitive inward currents more effectively in capsaicin insensitive $(61 \%)$ than sensitive neurons $(20 \%)$. These data demonstrate that the somata of different types of cultured sensory neurons have distinct mechanosensitive phenotypes that retain the properties associated with nerve terminal mechanosensors. ${ }^{9}$

Recently, a case for TRP-like channels as mechanosensors has been made. In particular TrpV4, a channel that is activated by stretch and heat has been found to be necessary for mechanical transduction of noxious pressure through studies of null mutant mice. ${ }^{10}$ It is not as yet clear whether this channel is involved in mechanosensation in visceral afferents

\section{SODIUM CHANNELS AS VISCERAL PAIN TARGETS}

The ten functional voltage gated sodium channels (VGSC) present in both the peripheral and central nervous system comprise a large membrane spanning $\alpha$ subunit of $260 \mathrm{kDa}$, which contains four repeated domains of six transmembrane segments. In addition, there are associated regulatory

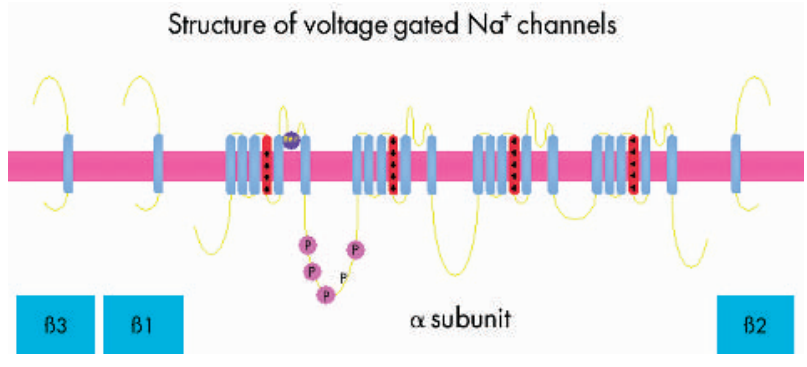

Figure 2 Structure of a voltage sodium channel $\alpha$ subunit showing the voltage sensor S4 domains and the accessory subunits that anchor the channel with respect to extracellular signals.

subunits-a $\beta_{1}$ subunit of $36 \mathrm{kDa}$ and a covalently associated $\beta_{2}$ subunit of $33 \mathrm{kDa}$. There is indirect evidence that a number of different $\beta 2$ subunits exist. The $\alpha$ subunit mRNAs can direct the translation of functional channels. However, the accessory $\beta_{1}$ and $\beta_{2}$ subunits enhance functional channel expression in Xenopus oocytes and regulate the kinetic properties of expressed channels. In addition, the $\beta$ subunits may play a role in anchoring the protein at particular locations within the cell.

The voltage gated sodium channel $\alpha$ subunit SNS Nav1.8 $8^{11}$ is particularly interesting, as it corresponds to an unusual type of sodium channel present in small diameter sensory neurons that is resistant to the puffer fish poison, tetrodotoxin (TTX). Mounting evidence suggests that the TTXr sodium channel plays a unique role in the transmission of nociceptive information to the spinal cord. ${ }^{12}$ The sodium channel transcripts present in dorsal root ganglia have been explored by Northern blots and PCR. The neuronal forms type I and II are present, whereas the embryonic type III reappears after axotomy. Both Navl.8 and the TTXs channel, Navl.7, are present at high levels in peripheral neurons. Navl.7, Navl.1, Navl.6, and Navl.2 TTX sensitive transcripts occur in descending order of abundance. The atypical sodium channel $\mathrm{NaG}$ is expressed predominantly by Schwann cells, but is also found in sensory neurons. Only the small diameter sensory neuron specific Navl.8 subunit is exclusively present in small diameter sensory neurons, however. This pattern of distribution has been demonstrated by in situ hybridisation and RTPCR examination of a range of tissues. In addition, the destruction of small diameter sensory neurons by capsaicin, which acts predominantly on nociceptors, leads to loss of the Nav1.8 transcript. ${ }^{11}$ These observations combined with the resistance to TTX block ( $\left.\mathrm{IC}_{50} 60 \mu \mathrm{M}\right)$ support the view that Nav1.8 underlies the TTXi currents observed in C fibres and small diameter neurons.

Prostaglandin $\mathrm{E}_{2}$, adenosine, and serotonin all increased the magnitude of Navl.8, shifted its conductance voltage relation in a hyperpolarising direction, and increased the rates of activation and inactivation of sodium channels in small diameter sensory neurons in culture. Such data suggest that sodium currents encoded by Navl.8 play an important role in regulating pain thresholds. Interestingly gastric inflammation is also able to recapitulate these effects. ${ }^{13}$

Recently a sodium channel transcript named $\mathrm{NaN}$ or Nav1.9 has been identified in small diameter sensory neurons. The channel contains the appropriate sodium selectivity filters and voltage sensor motifs, although it has three less positively charged residues in its S4 domains than SNS. Very interestingly the channel expresses a serine residue at the same position of the TTX binding site as SNS, suggesting that the channel is TTX resistant. There is evidence that the transcript encodes a functional channel that plays an important role in setting thresholds of 
activation of small diameter sensory neurons. ${ }^{14}$ In addition, Navl.9 but not Navl.8 has been found to be present at high levels in enteric neurons. ${ }^{15}$ Behavioural studies of Nav1.8 null mutant mice have provided compelling evidence that aspects of acute and inflammatory pain applied to the skin are deficient in the null mutant. ${ }^{11}$ Recent studies of a variety of models of colitis ${ }^{16}{ }^{17}$ suggest that the Navl.8 channel also plays an important role in the development of visceral hyperalgesia.

\section{CONCLUSIONS}

In order to use advances in microarray technology, and molecular genetics to catalogue the repertoire of receptors and channels expressed by visceral afferents, a great deal of basic labelling and analysis needs to be carried out. At present, no unique markers for visceral afferents are known. It does seem clear, however, that many aspects of nociceptor activation are common to visceral and other primary afferents, although the central consequences of activation seem to be different. Once gene ablation can be carried out selectively in visceral afferent neurons using the Cre-lox-p system, it should be possible to test the roles of individual receptors in the development of visceral pain.

\section{ACKNOWLEDGEMENTS}

The author thanks the MRC and the Wellcome Trust for their support.

\section{REFERENCES}

1 Wood JN. The Molecular Basis of Pain Induction. New York: John Wiley, 2000.

2 Caterina MJ, Schumacher MA, Tominaga $M$, et al. The capsaicin receptor: a heat-activated ion channel in the pain pathway. Nature 1997;389:816-24.
3 Peier A, Mogrich A, Hergarden A, et al. A TRP channel that senses cold stimuli and menthol. Cell 2002;108:705-15.

4 McKemy DD, Neuhausser WM, Julius D. Identification of a cold receptor reveals a general role for TRP channels in thermosensation. Nature 2002;416:52-8

5 Story GM, Peier AM, Reeve AJ, et al. ANKTM1, a TRP-like channel expressed in nociceptive neurons, is activated by cold temperatures. Cell 2003;112:819-29.

6 Kellenberger S, Schild L. Epithelial sodium channel/degenerin family of ion channels: a variety of functions for a shared structure. Physiol Rev 2002;82:735-67

7 Nakamura F, Strittmatter SM. P2Y1 purinergic receptors in sensory neurons: contribution to touch-induced impulse generation. Proc Natl Acad Sci USA 1996;93:10465-70.

8 Price MP, Mcllwrath SL, Xie J, et al. The DRASIC cation channel contributes to the detection of cutaneous touch and acid stimuli in mice. Neuron 2001;32:1071-83.

9 Drew LJ, Wood JN, Cesare P. Distinct mechanosensitive properties of capsaicin-sensitive and -insensitive sensory neurons. J Neurosci 2002;22:RC228.

10 Suzuki M, Mizuno A, Kodaira K, et al. Impaired pressure sensation with mice lacking TRPV4. J Biol Chem 2003;278:22664-8.

11 Akopian AN, Sivilotti L, Wood JN. A tetrodotoxin-resistant sodium channel expressed by C-fibre associated sensory neurons. Nature 1996:379:257-62.

12 Akopian AN, Souslova V, England S, et al. The tetrodotoxin-resistant sodium channel SNS plays a specialised role in pain pathways. Nat Neurosci 1999;2:541-8.

13 Bielefeldt K, Ozaki N, Gebhart GF. Experimental ulcers alter voltage-sensitive sodium currents in rat gastric sensory neurons. Gastroenterology 2002; 122:394-405.

14 Baker MD, Chandra SY, Ding Y, et al. GTP-induced tetrodotoxin-resistant $\mathrm{Na}+$ current regulates excitability in mouse and rat small diameter sensory neurones. J Physiol 2003:548:373-82.

15 Rugiero F, Mistry M, Sage D, et al. Selective expression of a persistent tetrodotoxin-resistant $\mathrm{Na}$ + current and $\mathrm{NaV} 1.9$ subunit in myenteric sensory neurons. J Neurosci 2003;23:2715-25.

16 Laird JM, Martinez-Caro L, Garcia-Nicas E, et al. A new model of visceral pain and referred hyperalgesia in the mouse. Pain 2001;92:335-42.

17 Laird JM, Souslova V, Wood JN, et al. Deficits in visceral pain and referred hyperalgesia in Nav1.8 (SNS/PN3)-null mice. J Neurosci 2002;22:8352-6. 